\title{
Bose-Einstein condensate collapse: A comparison between theory and experiment
}

\author{
C. M. Savage,* N. P. Robins, and J. J. Hope \\ Department of Physics and Theoretical Physics, Australian National University, Australian Capital Territory 0200, Australia
}

(Received 5 July 2002; published 28 January 2003)

\begin{abstract}
We solve the Gross-Pitaevskii equation numerically for the collapse induced by a switch from positive to negative scattering lengths. We compare our results with experiments performed with Bose-Einstein condensates of ${ }^{85} \mathrm{Rb}$, in which the scattering length was controlled using a Feshbach resonance. Building on previous theoretical work we identify quantitative differences between the predictions of mean-field theory and the results of the experiments. In addition to the previously reported difference between the predicted and observed critical atom number for collapse, we also find that the predicted collapse times systematically exceed those observed experimentally.

DOI: 10.1103/PhysRevA.67.014304

PACS number(s): 03.75.Hh, 03.75.Be
\end{abstract}

Most experiments on dilute gas Bose-Einstein condensates (BECs) are performed with atoms that have a repulsive two-body interaction. Exceptions are the experiments on ${ }^{7} \mathrm{Li}$ $[1,2]$ and, more recently, on ${ }^{85} \mathrm{Rb}[3,4]$. For ${ }^{85} \mathrm{Rb}$ a Feshbach resonance allows the two-body interaction strength to be tuned over a wide range of attractive and repulsive values. In particular, the scattering length has been rapidly switched from positive (repulsive interaction) to negative (attractive interaction) values, leading to the collapse and subsequent explosion of the condensate. Recently, the large positive scattering lengths attainable in this system have been used to produce atom-molecule condensates [5].

In the following we report on our modeling of the ${ }^{85} \mathrm{Rb}$ collapse experiments [3], using the Gross-Pitaevskii (GP) equation for the expectation value of the field operator [6-9]. Saito and Ueda [10], Adhikari [11,12], and Santos and Shlyapnikov [13] have also modeled these experiments by numerical solution of the cylindrically symmetric GP equation. Saito and Ueda concluded that this describes the collapsing and exploding dynamics at least qualitatively [10]. Following their suggestion, we report a more quantitative comparison between the theoretical and experimental results, and find significant differences.

The series of experiments on the collapse and explosion of ${ }^{85} \mathrm{Rb}$ BECs challenges theoretical models in a number of ways [14]. A body of theoretical work based on the GP equation predicts the critical number of atoms $N_{\mathrm{cr}}$ for collapse to be significantly larger than is observed. The expression for the critical number is

$$
N_{\mathrm{cr}}=k \frac{a_{\mathrm{ho}}}{|a|}
$$

where $a_{\mathrm{ho}}=\sqrt{\hbar /(m \bar{\omega})}$ is the harmonic oscillator scale length, with $\bar{\omega}$ the geometric mean of the trap frequencies in the three Cartesian directions, and $a$ is the scattering length. Experimentally, $k=0.46 \pm 0.06$ [4], whereas $k=0.57$ for spherically symmetric solutions of the GP equation $[15,16]$,

\footnotetext{
*Electronic address: craig.savage@anu.edu.au
}

and $k=0.55$ for cylindrically symmetric solutions [17] using the experimental parameters of the ${ }^{85} \mathrm{Rb}$ collapse experiments [4].

We have confirmed these GP predictions for the experimental case with cylindrically symmetric numerical solutions. We also performed full three-dimensional numerical solutions, and found that slight departures from cylindrical symmetry had no effect on the critical number [18]. Consequently, there is a disagreement near the two standard deviation level, which should be regarded as significant.

We also report a quantitative discrepancy between the predictions of the GP model and experiment. Under certain conditions, the GP predicted time to the initiation of collapse, $t_{\text {collapse }}$, is systematically longer than that observed in the experiments [3].

Finally, we discuss the possiblility that these discrepancies result from quantum field effects beyond the GP approximation. We therefore now derive the GP equation from the quantum field theory.

The second-quantized Hamiltonian for a dilute gas, in terms of the field operator $\hat{\Psi}(\mathbf{r}, t)$, is

$$
H=\int d \mathbf{r} \hat{\Psi}^{\dagger} H_{0} \hat{\Psi}+\frac{1}{2} \int d \mathbf{r} d \mathbf{r}^{\prime} \hat{\Psi}^{\dagger} \hat{\Psi}^{\dagger^{\prime}} V\left(\mathbf{r}-\mathbf{r}^{\prime}\right) \hat{\Psi}^{\prime} \hat{\Psi}
$$

where $\hat{\Psi}^{\prime}=\hat{\Psi}\left(\mathbf{r}^{\prime}, t\right)$ and $H_{0}$ is the single particle Hamiltonian for the kinetic energy and trapping potential,

$$
H_{0}=-\frac{\hbar^{2}}{2 m} \nabla^{2}+\frac{1}{2} m\left(\omega_{x}^{2} x^{2}+\omega_{y}^{2} y^{2}+\omega_{z}^{2} z^{2}\right),
$$

where $m$ is the atomic mass $\left(1.41 \times 10^{-25} \mathrm{~kg}\right.$ for $\left.{ }^{85} \mathrm{Rb}\right)$, and $\omega_{i}$ is the trap frequency along Cartesian axis $i$. In the limit of particles separated by distances much greater than the scattering length $a$ we approximate the two-body potential by a $\delta$ function interaction [6-9]

$$
V\left(\mathbf{r}-\mathbf{r}^{\prime}\right)=g \delta\left(\mathbf{r}-\mathbf{r}^{\prime}\right), \quad g=\frac{4 \pi \hbar^{2} a}{m} .
$$

The Heisenberg dynamical equation for the field operator is then 


$$
i \hbar \frac{\partial}{\partial t} \hat{\Psi}=H_{0} \hat{\Psi}+g \hat{\Psi}^{\dagger} \hat{\Psi} \hat{\Psi}
$$

Taking the symmetry-breaking approach we assume that the field expectation value is not zero and define it as the GP wave function $\langle\hat{\Psi}(\mathbf{r}, t)\rangle=\Phi(\mathbf{r}, t)$, normalized to the number of particles $N$,

$$
N=\int|\Phi(\mathbf{r}, t)|^{2} d \mathbf{r}
$$

Then taking the expectation value of the Heisenberg equation (5) gives

$$
i \hbar \frac{\partial}{\partial t} \Phi=H_{0} \Phi+g\left\langle\hat{\Psi}^{\dagger} \hat{\Psi} \hat{\Psi}\right\rangle .
$$

If we assume that the expectation value factorizes, as it would, for example, if the system were in an eigenstate of the field operator,

$$
\left\langle\hat{\Psi}^{\dagger} \hat{\Psi} \hat{\Psi}\right\rangle=\Phi^{*} \Phi \Phi
$$

then we obtain the GP equation

$$
i \hbar \frac{\partial}{\partial t} \Phi=\left(H_{0}+g|\Phi|^{2}\right) \Phi .
$$

In order to model atom loss due to three-body recombination we add a phenomenological term proportional to the density squared $|\Phi|^{2}$ with rate coefficient $K_{3} / 2$ [8]:

$$
i \hbar \frac{\partial}{\partial t} \Phi=\left(H_{0}+g|\Phi|^{2}-i \frac{\hbar}{2} K_{3}|\Phi|^{4}\right) \Phi .
$$

Note that Roberts et al. [19] use the noncondensed atom $K_{3}$, which must be divided by 3 ! to get our condensate $K_{3}$. We assume one-body and two-body loss are negligible, as was true for the relevant experiments. The number of atoms then decays as

$$
\frac{d N}{d t}=-K_{3} \int|\Phi(\mathbf{r}, t)|^{6} d \mathbf{r} .
$$

As an example of the ability of the GP equation to correctly model the ${ }^{85} \mathrm{Rb}$ [3] experiments we present Fig. 1. It is the result of a numerical solution of the (two-dimensional) cylindrically symmetric GP equation for $\Phi(r, z)$,

$$
\begin{aligned}
i \hbar \frac{\partial}{\partial t} \widetilde{\Phi}= & -\frac{\hbar^{2}}{2 m}\left(\partial_{r}^{2}+r^{-1} \partial_{r}+\partial_{z}^{2}\right) \widetilde{\Phi}+\frac{1}{2} m\left(\omega_{r}^{2} r^{2}+\omega_{z}^{2} z^{2}\right) \widetilde{\Phi} \\
& +g|\widetilde{\Phi}|^{2} \widetilde{\Phi}-i \frac{\hbar}{2} K_{3}|\widetilde{\Phi}|^{4} \widetilde{\Phi}
\end{aligned}
$$

Parameters are the same as those of Fig. 1(b) of Donley et al. [3]. Specifically, the ground state of the GP equation for $a$ $=+7 a_{0}$ was switched in $1 \mathrm{~ms}$ to $a=-30 a_{0}$, where $a_{0}$ $=0.0529 \mathrm{~nm}$ is the Bohr radius. For the three-body recombination rate coefficient $K_{3}=190 \times 10^{-28} \mathrm{~cm}^{6} \mathrm{~s}^{-1}$ the agree-

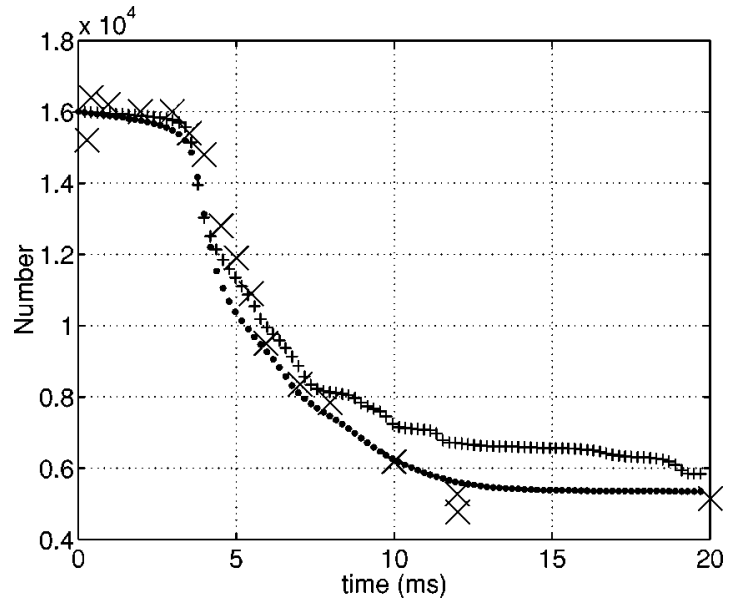

FIG. 1. Experimental and numerical results for the number of atoms $N$ versus time after a switch from $a=+7 a_{0}$ to $a=-30 a_{0}$. The experimental points $(X)$ are from Fig. 1(b) of Donley et al. [3]. The numerical results are for $K_{3}=190 \times 10^{-28} \mathrm{~cm}^{6} \mathrm{~s}^{-1}$ (filled circles) and for $K_{3}=78 \times 10^{-28} \mathrm{~cm}^{6} \mathrm{~s}^{-1}(+)$. Other parameters are as given in the experimental paper [3]: $N_{0}=16000$, radial frequency $\omega_{r}=2 \pi \times 17.5 \mathrm{~Hz}$, axial frequency $\omega_{z}=2 \pi \times 6.8 \mathrm{~Hz}$.

ment with the experimental results is good. However, it should be noted that the experimental points are the "remnant" atom number, while the numerical points are the total atom number, which overestimates the remnant atom number. A smaller value of $K_{3}$ agrees better with the earlier points, while overestimating the final atom number. The precise value of $K_{3}$ has little effect on the conclusions of this paper, which concern the initiation of collapse.

These results agree with those reported by Saito and Ueda [10], Adhikari [11,12], and Santos and Shlyapnikov [13]. However Saito and Ueda [10] used a much smaller value of the three-body recombination rate coefficient $K_{3}=2$ $\times 10^{-28} \mathrm{~cm}^{6} \mathrm{~s}^{-1}$. This produces the collapses and revivals in condensate size that were observed in their simulations. These only become important for $K_{3}$ less than about $10^{-26} \mathrm{~cm}^{6} \mathrm{~s}^{-1}$. Since three-body recombination is responsible for the atom loss, it is remarkable that such a wide range of coefficients reproduces the experimental results.

The three-body recombination rate coefficient $K_{3}$ is expected to vary strongly near the Feshbach resonance [20]. Experimental determination of $K_{3}$ is difficult due to the low densities of ${ }^{85} \mathrm{Rb}$ condensates. Upper bounds have been estimated to be $5 \times 10^{-25} \mathrm{~cm}^{6} \mathrm{~s}^{-1}$, dropping to $10^{-26} \mathrm{~cm}^{6} \mathrm{~s}^{-1}$ nearer the Feshbach resonance [19]. Far from resonance it has been measured to be $(4.24 / 3 !) \times 10^{-25} \mathrm{~cm}^{6} \mathrm{~s}^{-1}=7.1$ $\times 10^{-26} \mathrm{~cm}^{6} \mathrm{~s}^{-1}$ [19].

The cylindrically symmetric numerical simulations were performed on a $512 \times 512$ grid, $35.64 \mu \mathrm{m}$ long in the axial (z) direction and with the radial coordinate extending to $11.88 \mu \mathrm{m}$. The corresponding spatial grid spacings were therefore $0.07 \mu \mathrm{m}$ and $0.023 \mu \mathrm{m}$. The time steps were 2.34 ns. All simulations were performed on a multiprocessor machine [21], using up to 32 processors, and the RK4IP algorithm developed by the BEC theory group of R. Ballagh at the University of Otago [22]. This is a pseudospectral method with a Runge-Kutta time step. The cylindrically 


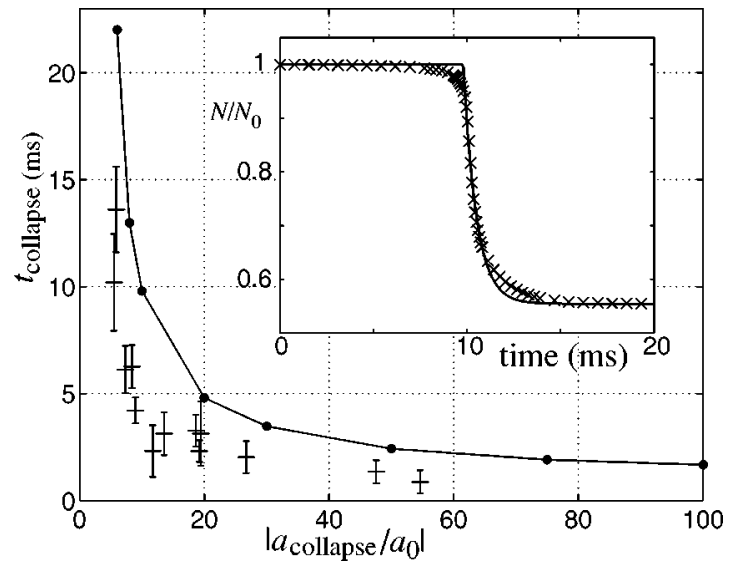

FIG. 2. Experimental and numerical results for the collapse time $t_{\text {collapse }}$ versus scattering length $a_{\text {collapse }}$ after a switch from $a=0$ to $a_{\text {collapse }}$. The experimental points $(+)$ and their error bars are from Fig. 2 of Donley et al. [3]. The numerical results (filled circles) are for $K_{3}=190 \times 10^{-28} \mathrm{~cm}^{6} \mathrm{~s}^{-1}$. Other parameters are as given in the Fig. 1 caption, except for $N_{0}=6000$. Inset: example of the fitting procedure used to determine the collapse times. Shown is a fit of the functional form Eq. (13) (solid line) to the GP simulation $(X)$ for $a=-10 a_{0}$. The fit parameters here are $t_{\text {collapse }}=9.8 \mathrm{~ms}, \tau_{\text {decay }}$ $=0.7 \mathrm{~ms}$, and $N_{\mathrm{f}} / N_{0}=0.5544$.

symmetric and full three-dimensional codes are independent and were cross checked. Grid spacings and time steps were varied to ensure convergence. Overall the results were found to be quite robust. As another test, we solved the GP equation for one-half a radial period after the quenching of the collapse. As was observed experimentally, the condensate refocused onto the axis, due to the oscillation in the harmonic radial potential. All this, together with the agreement of our results with those of Saito and Ueda [10], Adhikari [11,12], and Santos and Shlyapnikov [13], gives us confidence in their accuracy. Following Adhikari [11], the initial condition for Fig. 1 was generated by adiabatically expanding the harmonic oscillator initial state $a=0$ to $a=+7 a_{0}$ over $444 \mathrm{~ms}$.

Figure 2 presents our calculations of the collapse times $t_{\text {collapse }}$ for the conditions of Fig. 2 of Donley et al. [3]. They are consistent with the simulation results reported in Fig. 3 of [10], Fig. 4, of [12], and Fig. 2 of [13]. The collapse times were determined by visually fitting plots of atom number versus time to the functional form

$$
N=\left(N_{0}-N_{\mathrm{f}}\right) \exp \left[-\left(t-t_{\text {collapse }}\right) / \tau_{\text {decay }}\right]+N_{\mathrm{f}},
$$

where $N_{\mathrm{f}}$ is the long time atom number. An example is given in the inset to Fig. 2. We have also plotted the experimental results reported in Fig. 2 of Donley et al. [3], and find a small, but significant, systematic disagreement with the GP results. Although the reported errors in the experimental collapse times are large, the GP values for $t_{\text {collapse }}$ are consistently longer than the experimental ones. This is surprising as the GP model is expected to be valid for the low densities preceding the collapse. If it were to fail, it would be expected to do so at the high densities generated subsequently. Never- theless, the disagreement is not unprecedented since, as we discussed earlier, the GP model also overestimates the critical number for collapse.

The estimates of $t_{\text {collapse }}$ by Saito and Ueda [10] (their Fig. 3) are between 5\% (low $a$ ) and 10\% (high $a$ ) smaller than ours. This is consistent with the smaller three-body recombination rate coefficient $K_{3}$ they used. However, their results are still significantly longer than the experimentally measured times.

We have confirmed these cylindrically symmetric simulations by performing full three-dimensional simulations. In particular we broke the cylindrical symmetry by using trap frequencies of $17.24 \times 17.47 \times 6.80 \mathrm{~Hz}$ [4].

We were unable to substantially improve the agreement either by changing the initial condition to reflect the experimental uncertainty of $a= \pm 2 a_{0}$, or by varying the threebody recombination rate coefficient. This suggests that some of the physics determining the collapse time is not captured by our GP model.

Both the collapse time and critical number discrepancies could be resolved by using a scattering length in the GP model larger in magnitude than the experimental value. This would reduce the collapse time and decrease the critical number, as required. The required increases in the scattering length magnitudes vary, ranging from a factor of $0.57 / 0.46$ $=1.2$ for the critical number, up to a factor of about 2 for the collapse times for large $a_{\text {collapse }}$. However, the scattering length is experimentally well calibrated [23], so any such change would reflect a deficiency of our GP model.

One possible origin of the discrepancy is the effect of thermal noncondensed atoms. Because of the quantum statistics of collisions between bosons, the interaction potential between a condensed atom and an atom in another mode is twice that between two condensed atoms. Hence one might expect the presence of thermal uncondensed atoms to shorten the collapse time compared to the GP prediction, as observed. Furthermore, this might be approximately corrected for by using an increased magnitude effective scattering length in the GP model. However, the uncondensed fraction is much less than $10 \%$ of the total number of atoms [14], so it seems unlikely that this effect is large enough to account for the discrepancy. Furthermore, Roberts et al. [4] reported that the critical number for collapse $N_{\text {cr }}$ was insensitive to varying the temperature. Therefore we do not expect finite temperature extensions of the GP theory to explain the discrepancy [24].

Another possible origin is the formation of ${ }^{85} \mathrm{Rb}$ molecules, which is ignored in our model. This is justified because the scattering lengths for which molecular formation has been found to be important [25,26] are $a=2500 a_{0}$ or higher. The highest magnitude we have considered, $a=$ $-100 a_{0}$, is more than an order of magnitude smaller. Consequently, we consider it unlikely that molecule formation is important for the initiation of collapse.

The possible origin we favor is quantized atom field effects. These might arise due to a breakdown of the factorization assumption Eq. (8). There have been several suggestions for how the quantized field might influence the collapse [2729 ], including a prediction of squeezing of vacuum fluctua- 
tions by the collapsing condensate [30]. Furthermore, Nozières [31] has emphasized that only for positive scattering lengths does an energy barrier protect BECs from fragmentation into many populated states [7]. For negative scattering lengths, mean-field energy is released when atoms scatter from the condensate into other modes. Fragmentation might be approximated by increasing the effective two-body interaction potential in the GP model by up to a factor of 2 . This might explain why the experimentally observed collapse times and critical numbers are smaller than predicted by mean-field theory.

In order to investigate the behavior of a fully quantized atom field, we have used the gauge- $P$ function approach recently developed by Deuar and Drummond [32]. This method overcomes some of the problems that plague stochastic simulations based on the positive $P$-function quasiprobability distribution $[33,34]$. We were computationally limited to simulations in one spatial dimension and found agreement with the GP collapse times at the 1\% level. Although this preliminary work does not provide evidence for quantum field effects, it is important to extend the fully quantized field modeling to three spatial dimensions, and hence to use actual experimental parameters.

In conclusion, although our simulation results agree with those of previous authors, we have reached the conclusion that there is a significant discrepancy between the predictions of the GP model and the experimental measurements. This suggests a reconsideration of modeling of ${ }^{85} \mathrm{Rb}$ BEC collapse experiments.

This research was supported by the Australian Research Council and by the National Facility of the Australian Partnership for Advanced Computing [21].
[1] C.C. Bradley, C.A. Sackett, and R.G. Hulet, Phys. Rev. Lett. 78, 985 (1997).

[2] J.M. Gerton et al., Nature (London) 408, 692 (2000).

[3] E.A. Donley et al., Nature (London) 412, 295 (2001).

[4] J.L. Roberts et al., Phys. Rev. Lett. 86, 4211 (2001).

[5] E.A. Donley et al., Nature (London) 417, 529 (2002).

[6] F. Dalfovo et al., Rev. Mod. Phys. 71, 463 (1999).

[7] A.J. Leggett, Rev. Mod. Phys. 73, 307 (2001).

[8] C.J. Pethick and H. Smith, Bose-Einstein Condensation in Dilute Gases (Cambridge University Press, Cambridge, England, 2002).

[9] P. Meystre, Atom Optics (Springer-Verlag, New York, 2001).

[10] H. Saito and M. Ueda, Phys. Rev. A 65, 033624 (2002).

[11] S.K. Adhikari, Phys. Lett. A 296, 145 (2002).

[12] S.K. Adhikari, Phys. Rev. A 66, 013611 (2002); 66, 043601 (2002).

[13] L. Santos and G.V. Shlyapnikov, Phys. Rev. A 66, 011602 (2002).

[14] N.R. Claussen et al., Phys. Rev. Lett. 89, 010401 (2002).

[15] A. Eleftheriou and K. Huang, Phys. Rev. A 61, 043601 (2000).

[16] P.A. Ruprecht et al., Phys. Rev. A 51, 4704 (1995).

[17] A. Gammal, T. Frederico, and L. Tomio, Phys. Rev. A 64, 055602 (2001).

[18] These numerical solutions were performed on spatial grids as large as $512 \times 256 \times 256$.

[19] J.L. Roberts et al., Phys. Rev. Lett. 85, 728 (2000).
[20] B.D. Esry, C.H. Greene, and J.P. Burke, Jr., Phys. Rev. Lett. 83, 1751 (1999).

[21] Details of the machine are given on the web site of the National Faciltity of the Australian Partnership for Advanced Computing: http://nf.apac.edu.au/.

[22] The RK4IP method is described in B.M. Caradoc-Davies, Ph.D. thesis, University of Otago, Dunedin, New Zealand (2000); http://www.physics.otago.ac.nz/bec2/bmcd/.

[23] J.L. Roberts et al., Phys. Rev. Lett. 81, 5109 (1998).

[24] M.J. Davis, R.J. Ballagh, and K. Burnett, J. Phys. B 34, 4487 (2001).

[25] E.A. Donley et al., Nature (London) 417, 529 (2002).

[26] S.J.J.M.F Kokkelmans and M.J. Holland, Phys. Rev. Lett. 89, 180401 (2002).

[27] M. Ueda and A.J. Leggett, Phys. Rev. Lett. 80, 1576 (1998).

[28] R.A. Duine and H.T.C. Stoof, Phys. Rev. Lett. 86, 2204 (2001).

[29] V.A. Yurovsky, Phys. Rev. A 65, 033605 (2002).

[30] E.A. Calzetta and B.L. Hu, e-print cond-mat/0207289.

[31] P. Nozières, in Bose Einstein Condensation, edited by A.Griffin, D. W. Snoke, and S. Stringari (Cambridge University Press, New York, 1995).

[32] P. Deuar and P.D. Drummond, Phys. Rev. A 66, 033812 (2002).

[33] M.J. Steel et al., Phys. Rev. A 58, 4824 (1998).

[34] P.D. Drummond and J.F. Corney, Phys. Rev. A 60, R2661 (1999). 